\title{
Instabilities and Mixing in Type II-P and II-b Supernovae
}

\author{
By T. SHIGE Y A M A, ${ }^{1}$ K. I W A MO TO, ${ }^{1}$ I. HA CHIS U, ${ }^{1}$ \\ K. NOMOTO, ${ }^{1}$ AND H. SAIO ${ }^{2}$ \\ ${ }^{1}$ University of Tokyo, Bunkyo-ku, Tokyo 113, Japan \\ ${ }^{2}$ Tohoku University, Sendai 980, Japan
}

We calculate a nonlinear growth of the Rayleigh-Taylor instability in the exploding red supergiant stars with a two-dimensional hydrodynamical code, and examine how the extent of mixing depends on the progenitor's core mass and the envelope mass. The results are compared with the observations of type II-P supernovae and the recent type II-b supernova 1993J.

\section{Introduction}

Large scale mixing in supernova ejecta has been indicated in spectroscopic and photometric observations of various types of supernovae. This has stimulated $2 \mathrm{D}$ and $3 \mathrm{D}$ hydrodynamical calculations of the Rayleigh-Taylor (R-T) instabilities during supernova explosions for SN 1987A (Arnett et al. 1989; Hachisu et al. 1990, 1992; Fryxell et al. 1991; Müller et al. 1991; Den et al. 1990; Yamada et al. 1990; Yamada \& Sato 1991; Herant \& Benz 1991, 1992), type Ib/Ic supernovae (Hachisu et al. 1991, 1994a), type II-P supernovae (Herant \& Woosley 1994; Hachisu et al. 1994b), and the type II-b supernova 1993J (Iwamoto et al. 1994). In particular, Hachisu et al. (1991, 1994a) found that development of the R-T instabilities depend sensitively on the presupernova structure, so that the comparison between hydrodynamical simulations and observations can provide a new clue to the understanding of supernova progenitors, their structure, and the explosion mechanism.

In the present paper, we follow a nonlinear growth of the $R-T$ instabilities in the exploding red supergiant stars, i.e., type II-P and II-b supernovae. We find that the extent of mixing depends on the core mass and the envelope mass of red supergiants.

\section{Initial Models}

We adopt the red-supergiant progenitor models for type II-P and II-b supernovae (Nomoto \& Hashimoto 1988; Saio et al. 1988). Their main-sequence masses $M_{\mathrm{ms}}$, and their presupernova helium core masses $M_{\mathrm{He}}, \mathrm{H}$-rich envelope masses $M_{\mathrm{H}}$, and the radii $R$ at the explosions are summarized in table 1 . The assumed neutron star baryonic masses $M_{\mathrm{NS}}$ are also given, so that the ejected $\mathrm{H}$-free core masses are given by $M_{\mathrm{He}}-M_{\mathrm{NS}}$.

For type II-P models, no mass loss is assumed. The core of IIP20 progenitor model is the same as adopted for the model of SN 1987A except for the hydrogen-rich envelope (Shigeyama \& Nomoto 1990).

For type II-b models, large amount of mass loss is assumed so that almost all hydrogenrich envelopes are lost at presupernova stage (e.g., Nomoto et al. 1993; Shigeyama et al. 1994). 
TABLE 1. Progenitor models of type II-P and II-b supernovae

\begin{tabular}{lccccc}
\hline Model & $M_{\mathrm{ms}} / M_{\odot}$ & $M_{\mathrm{H}} / M_{\odot}$ & $M_{\mathrm{He}} / M_{\odot}$ & $M_{\mathrm{NS}} / M_{\odot}$ & $R / R_{\odot}$ \\
\hline IIP13 & 13 & 9.7 & 3.3 & 1.2 & 360 \\
IIP20 & 20 & 14 & 6 & 1.6 & 900 \\
IIb3H11 & 13 & $\mathbf{0 . 1 1}$ & 3.3 & $\mathbf{1 . 2}$ & 450 \\
IIb4H13 & 15 & $\mathbf{0 . 1 3}$ & 4 & $\mathbf{1 . 3}$ & 580 \\
\hline \hline
\end{tabular}

\section{Rayleigh-Taylor Instabilities and Mixing}

A shock wave is generated by depositing thermal energy at the mass cut that divides the ejecta and the neutron star. The final kinetic energy of explosion is assumed to be $E=1 \times 10^{51}$ erg for all the models. The early phase of shock propagation up to the $\mathrm{He} / \mathrm{C}+\mathrm{O}$ interface is calculated with a spherically symmetric Lagrangian code (Shigeyama \& Nomoto 1990). When the blast shock arrives at the bottom of the helium layer, the density, velocity, energy etc. are mapped onto our 2-D grids. Just after the mapping, we perturb only the velocity field all over the mesh points; i.e, the velocity field inside the shock front is perturbed but the velocities outside the shock remain zero.

We assume a random perturbation mode as in Hachisu et al. (1992). The amplitude of the perturbation is $5 \%$ of the expansion velocity. The mode of random perturbation is $n=128$ (i.e., the azimuthal angle is divided into 128 and the velocity is perturbed randomly at each divided azimuthal angle). The basic part of our code is based on Chakravarthy \& Osher's (1985) third-order accurate, TVD (Total Variation Diminishing) method (Hachisu et al. 1992).

\subsection{Type II-P Supernovae}

When the shock wave arrives at the composition interface, the expansion of the core is decelerated to generate a reverse shock. Such a deceleration induces the R-T instabilities which lead to mixing of materials. The nonlinear growth of the R-T instabilities for type II-P supernova explosions is shown in the density contours (left), which is linearly spaced by $5 \%$ of the maximum density, and the position of marker particles (right) in Figure 1. Marker particles, which are initially placed at each composition interface, follow the deformation of each composition interface, $\mathrm{H} / \mathrm{He}, \mathrm{He} / \mathrm{C}+\mathrm{O}$, and $\mathrm{O} / \mathrm{Si}+\mathrm{Ni}$. The elemental mixing in these type II-P models are plotted in Figure 2. It is found that the mixing of ${ }^{56} \mathrm{Ni}$ depends on the stellar mass, being substantial for the $\sim 13 M_{\odot}$ star, but much less for the $20 M_{\odot}$ star.

Model IIP20: The R-T instability is weak at the $\mathrm{He} / \mathrm{C}+\mathrm{O}$ interface as found in the model for SN 1987A (Hachisu et al. 1990, 1992), but it grows at the $\mathrm{H} / \mathrm{He}$ interface. The development of R-T instabilities in this II-P model is somewhat different from SN 1987A. Because of the lower envelope density and thus the larger density contrast at the $\mathrm{H} / \mathrm{He}$ interface, the instability grows faster and mix hydrogen well into the core material at the expansion velocity of as low as $1000 \mathrm{~km} \mathrm{~s}^{-1}$ (Figure 2a). The instability is so strong that the hydrogen, helium, and carbon-oxygen are well mixed even for a small amplitude of $1 \%$ and $0.2 \%$.

On the other hand, the propagation of the reverse shock is slower than in SN 1987A due to the lower envelope density. When the blast shock breaks out of the surface $\left(t \sim 3 \times 10^{5}\right.$ 

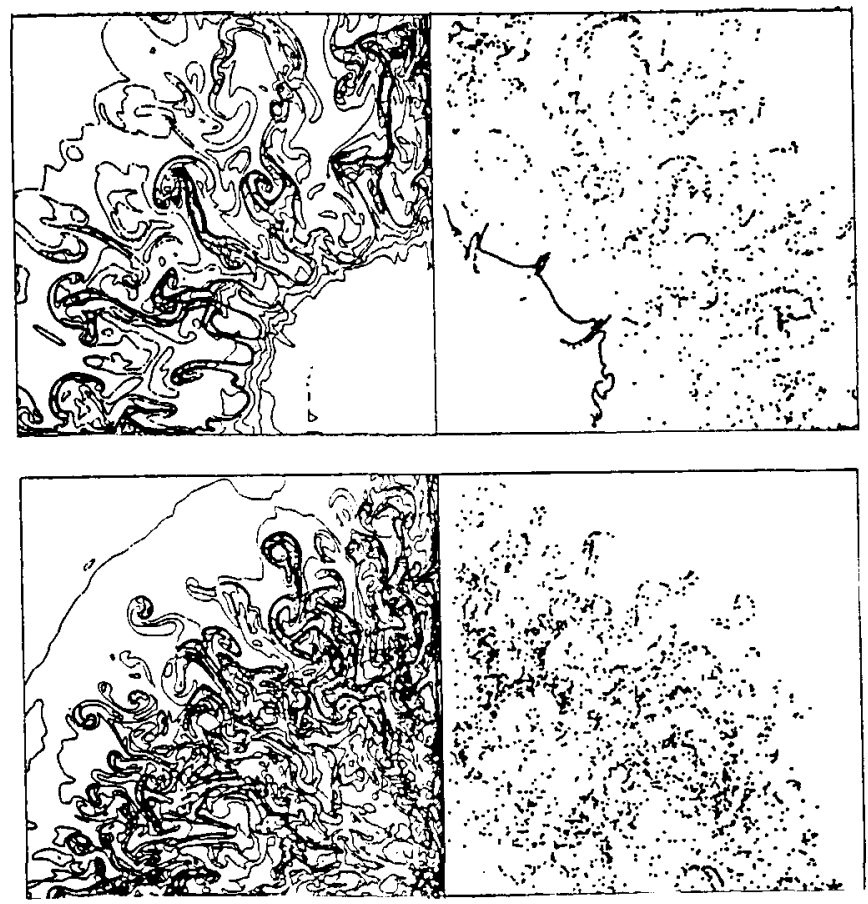

Figure 1. Density contour (left) and positions of marker particles (right) on $1025 \times 1025$ grids for type II-P supernova explosions. (a) IIP20 (upper): Time after the explosion is $8 \times 10^{5} \mathrm{~s}$. The size of the computational box is $1.65 \times 10^{14} \mathrm{~cm}\left(2400 R_{\odot}\right)$. (b) IIP13 (lower): Time after the explosion is $3 \times 10^{5} \mathrm{~s}$. The size is $6.8 \times 10^{13} \mathrm{~cm}\left(980 R_{\odot}\right)$.

s), the reverse shock just arrives at the $\mathrm{Si} / \mathrm{Ni}$ layer. After the shock breakout, the $\mathrm{R}-\mathrm{T}$ instability disappears quickly because a rarefaction wave causes the pressure inversion to disappear. Accordingly, the time is too short for the R-T instability to mix the innermost part of the ejecta, i.e., ${ }^{56} \mathrm{Ni}$ is not well mixed as seen from the marker particles at the $\mathrm{O} / \mathrm{Si}+\mathrm{Ni}$ interface (Fig. 1), which is a clear contrast to the model of SN 1987A.

Model IIP13: The R-T instability first grows at the $\mathrm{He} / \mathrm{C}+\mathrm{O}$ interface and then is amplified at the $\mathrm{H} / \mathrm{He}$ interface. Accordingly ${ }^{56} \mathrm{Ni}$ is well mixed into the middle of the hydrogen-rich envelope. Hydrogen is also well mixed down into the core. The elemental mixing is plotted in Figure 2b. Nickel is mixed up to the layer having the expansion velocity of $2000 \mathrm{~km} \mathrm{~s}^{-1}$ while hydrogen is mixed down to $500 \mathrm{~km} \mathrm{~s}^{-1}$.

\subsection{SN 1993J}

Figure 3 shows developments of the R-T instabilities as in Figure 1. For both models of $3 \mathrm{H} 11$ and $4 \mathrm{H} 13$, the R-T instability first grows at the $\mathrm{He} / \mathrm{C}+\mathrm{O}$ interface as in IIP13. For the smaller mass core, the instability is stronger because of larger deceleration due to larger mass ratio between the He-layer and the heavy element layer (Hachisu et al. 1991). The instabilities induce mixing of heavy elements (including ${ }^{56} \mathrm{Ni}$ ) into the helium layer. The extent of ${ }^{56} \mathrm{Ni}$ mixing is larger for the smaller mass helium core. It also depends on the degree of premixing of ${ }^{56} \mathrm{Ni}$ due to the $\mathrm{R}$ - $\mathrm{T}$ instability induced by neutrino heating (Herant et al. 1992). The $\mathrm{R}-\mathrm{T}$ instability at the $\mathrm{H} / \mathrm{He}$ interface also grows because of the large density jump across the core and the low density envelope. The instability is 

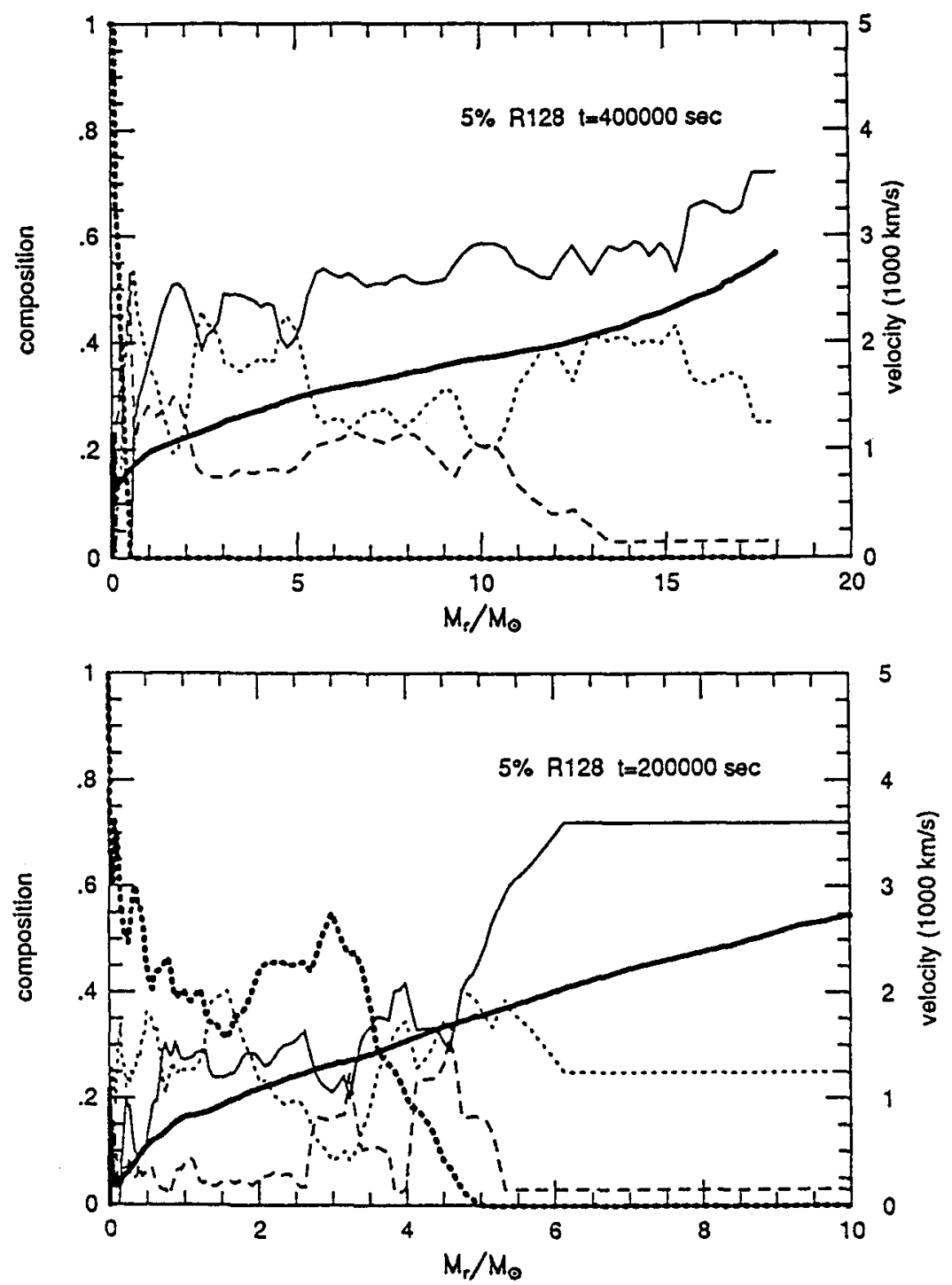

FIGURE 2. Elemental abundances are averaged along the angular direction and plotted against the mass coordinate, $M_{r}$, for the models in Figure 1 . Shown are $\mathrm{H}$ (thin solid), $\mathrm{He}$ (short-dashed), $\mathrm{C}+\mathrm{O}$ (long-dashed), and $\mathrm{Si}+{ }^{56} \mathrm{Ni}$ (thick-dashed). The averaged expansion velocity (thick solid) is also shown. (a) IIP20 (upper): $\mathrm{Ni}+\mathrm{Si}$ are localized near the center but hydrogen is well mixed into the core having an expansion velocity of $1000 \mathrm{~km} \mathrm{~s}^{-1}$. (b) IIP13 (lower): $\mathrm{Ni}+\mathrm{Si}$ are well mixed into the hydrogen-rich envelope. Conversely hydrogen is mixed down to the layer having an expansion velocity of $500 \mathrm{~km} \mathrm{~s}^{-1}$.

weaker than in type II-P supernova models and SN 1987A because the deceleration of the core expansion is weaker due to the much smaller envelope mass. However, a certain degree of mixing between the H-rich envelope and the helium layer is found.

Figure 4 shows the abundance distribution of $\mathrm{H}, \mathrm{He}, \mathrm{C}+\mathrm{O}$, and $\mathrm{Si}+\mathrm{Ni}$ for $3 \mathrm{H} 11$ in 2D and 1D calculations (i.e., with and without mixing) against the expansion velocity. Oxygen and ${ }^{56} \mathrm{Ni}$ are mixed up to the expansion velocity of $6000 \mathrm{~km} \mathrm{~s}^{-1}$ and $5300 \mathrm{~km}$ $\mathrm{s}^{-1}$, respectively. 

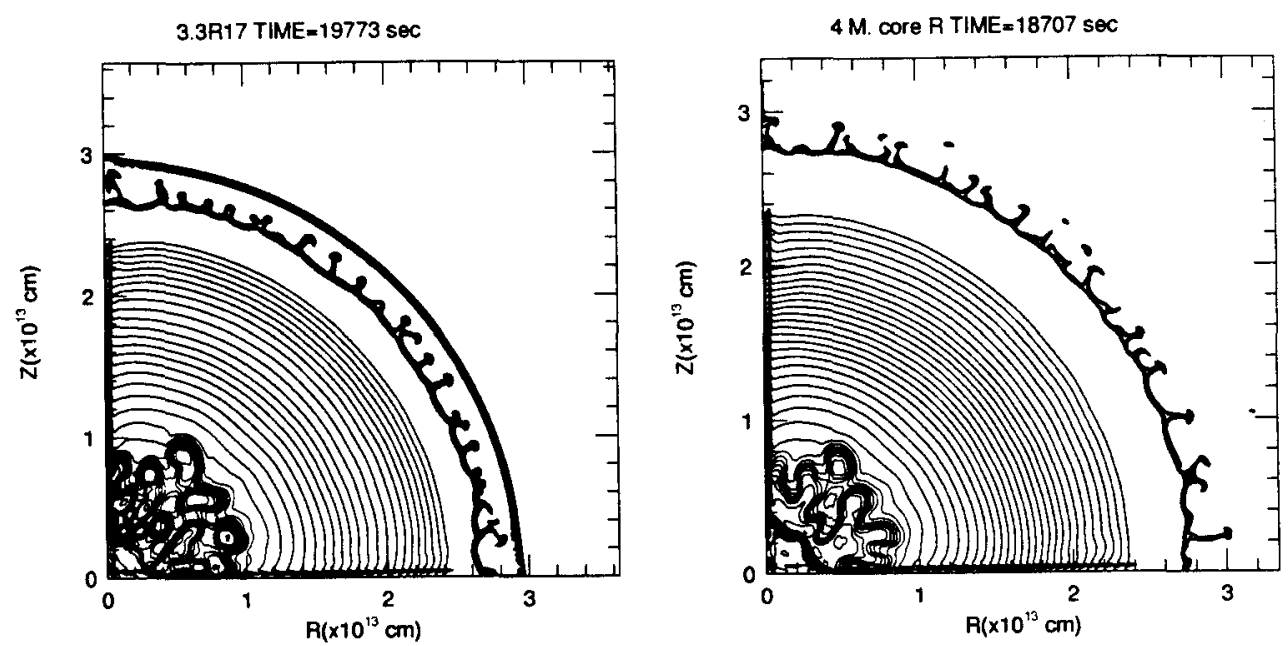

Figure 3. Developments of Rayleigh-Taylor instabilities in the models for the type II-b supernova SN 1993J: 4H13 (right) and $3 \mathrm{H} 11$ (left). Instabilities at both interfaces of $\mathrm{H} / \mathrm{He}$ and $\mathrm{He} / \mathrm{C}+\mathrm{O}$ are observed.

\section{Comparison with Observations}

Light curves of type II-P supernovae are characterized by the plateau which last typically $\sim 100$ day. The shape of the light curve is determined by the receding hydrogen recombination front in the ejecta (Grasberg et al. 1971). The plateau phase is longer as the minimum expansion velocity of hydrogen is lower, i.e., the mass of $\mathrm{H}$-rich envelope is larger and/or the mixing of hydrogen into the low velocity core is deeper (Shigeyama \& Nomoto 1990). Therefore, the length of the plateau may provide an indication of mixing as is the case of SN $1987 \mathrm{~A}$.

The mixing of ${ }^{56} \mathrm{Ni}$ also affect the light curve due to radioactive heating. Its extent is different between the above two progenitors: ${ }^{56} \mathrm{Ni}$ is mixed up to the middle of the hydrogen-rich envelope in the $13 M_{\odot}$, while it is hardly mixed for the $20 M_{\odot}$ stars.

The light curve of SN 1993J can be better reproduced with the above models if some ${ }^{56} \mathrm{Ni}$ is mixed into the helium layer. The extent of ${ }^{56} \mathrm{Ni}$ mixing found in these models is consistent with that suggested from the light curve. The small extent of hydrogen mixing is consistent with the relatively high hydrogen velocity in SN 1993J.

More direct indication of mixing in SN 1993J has been provided by the emission line features of oxygen (Wang \& Hu 1993; Spyromilio 1993) which indicate the presence of clumps. Since the instabilities at the $\mathrm{He} / \mathrm{C}+\mathrm{O}$ interface grows only for $M_{\mathrm{ms}}=13-$ $15 M_{\odot}$ but not for $M_{\mathrm{ms}} \sim 20 M_{\odot}$, such clumps in SN 1993J are consistent with the progenitor mass of $M_{\mathrm{ms}}=13-15 M_{\odot}$ as estimated from the light curve (Nomoto et al. 1993; Wheeler \& Filippenko 1994).

\section{Acknowledgements}

This work has been supported in part by the Grant-in-Aid for Scientific Research (04640265, 05242102, 05242103, 05242207) of the Japanese Ministry of Education, Science, and Culture, KEK, the Space Data Analysis Center of ISAS, and KiCFD. 


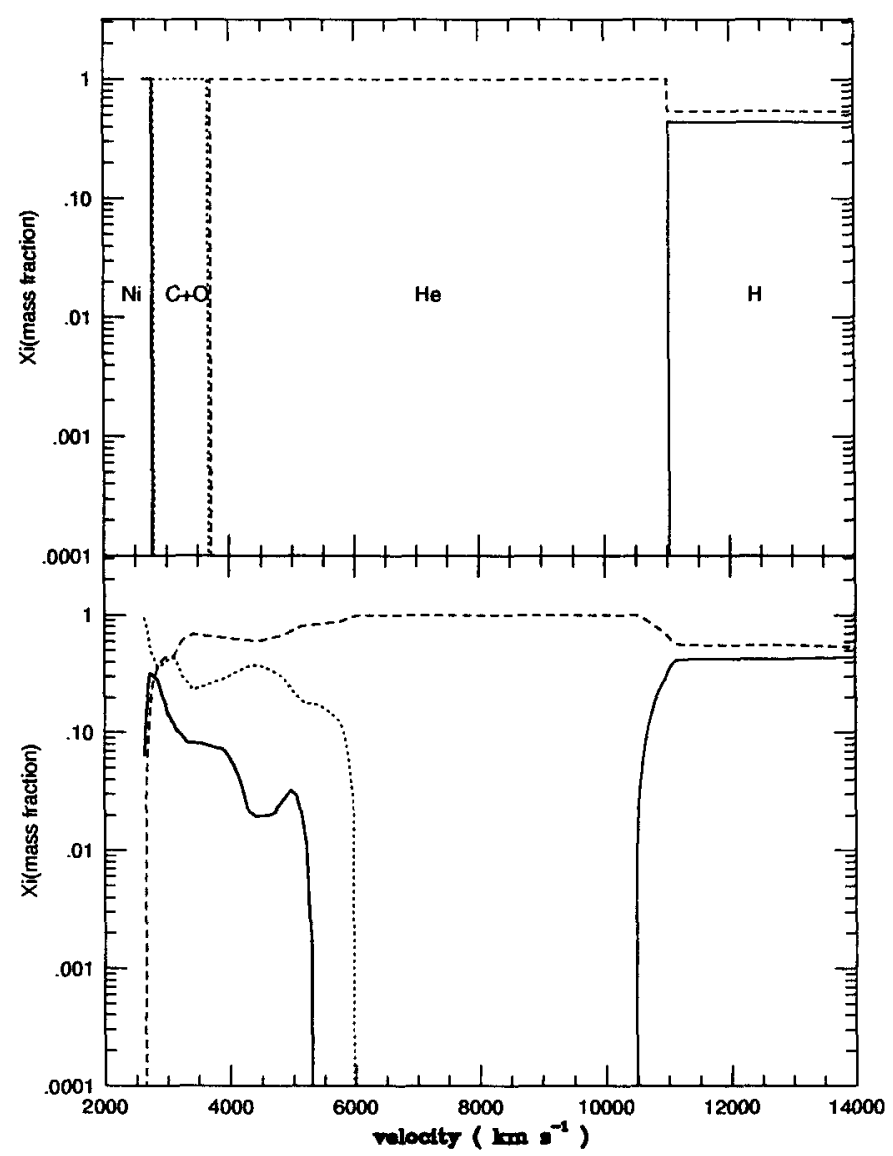

Figure 4. The abundance distribution of $\mathrm{H}, \mathrm{He}, \mathrm{C}+\mathrm{O}$, and $\mathrm{Si}+\mathrm{Ni}$ for $3 \mathrm{H} 11$ in $\mathrm{DD}$ and $2 \mathrm{D}$ calculations (i.e., with and without the mixing) against the expansion velocity.

\section{REFERENCES}

Arnett W.D., Fryxell B.A., \& Müller E. 1989, ApJ, 341, L63

Chakravarthy S.R., \& Osher S. 1985, AIAA Paper No. 85-0363

Den M., Yoshida T., \& Yamada Y. 1990, Progr. Theor. Phys., 83, 723

Fryxell B.A., Müller E., \& Arnett W.D. 1991, ApJ, 367, 619

Grasberg E.K., Imshennik, V.S., Nadezhin, D.K. 1971, Ap.Space Sci., 10, 28

Hachisu I., Matsuda T., Nomoto K., \& Shigeyama T. 1990, ApJ, 358, L57

Hachisu I., Matsuda T., Nomoto K., \& Shigeyama T. 1991, ApJ, 368, L27

Hachisu I., Matsuda T., Nomoto K., \& Shigeyama T. 1992, ApJ, 390, 230

Hachisu I., Matsuda T., Nomoto K., \& Shigeyama T. 1994a, A\&AS, in press

Hachisu, I., Nomoto, K., \& Shigeyama, T. 1994b, in ApJ, 390, 230 •

Herant M., \& Benz W. 1991, ApJ, 370, L81

Herant M., \& Benz W. 1992, ApJ, 387, 294

Herant M., Benz W. , \& Colgate, S. A. 1992, ApJ 395, 642

Herant M., \& Woosley, S.E. 1994, ApJ, 425, 814

Iwamoto, K., Hachisu, I., Shigeyama, T., \& Nomoto, K. 1994, in preparation

Müller E., Fryxell B., \& Arnett W.D. 1991, A\&A, 251, 505 
Nomoto, K., \& Hashimoto, M. 1988, Phys. Rep. 163, 13

Nomoto K., Shigeyama T., Kumagai S., Yamaoka H., \& Suzuki T 1993, in: Supernovae (Les Houches, Session LIV), ed. J. Audouze et al. , Elsevier Sci. Publ., in press

Nomoto, K., Suzuki, T., Shigeyama, T., Kumagai, S., Yamaoka, H., \& Saio, H. 1993, Nature, 364,507

Saio, H., Nomoto, K., \& Kato, M. 1988, Nature ,334, 508

Shigeyama, T., \& Nomoto, K. 1990, ApJ, 360, 242

Shigeyama, T., Nomoto, K., Tsujimoto, T \& Hashimoto, M. 1990, ApJ, 361, L23

Shigeyama, T., Suzuki, T., Kumagai, S., Nomoto, K., Saio, H., \& Yamaoka, H. 1994, ApJ, 420, 341

Wheeler, J.C., \& Filippenko, A.V. 1993, in this volume

Yamada Y., Nakamura T., \& Oohara K. 1990, Progr. Theor. Phys., 84, 436

Yamada S., \& Sato K. 1991, ApJ, 382, 594 